\title{
Constructing a Laplacian on the Diamond Fractal
}

\author{
Jun Kigami, Robert S. Strichartz, and Katharine C. Walker
}

\section{CONTENTS}

\section{Introduction}

2. Construction of Dirichlet Forms

3. Harmonic Functions

4. Existence Proof

Acknowledgements

References
Strichartz's research was supported in part by the National Science Foundation. Walker's research was supported by the National Science Foundation through the Research Experiences for Undergraduates (REU) Program.

\begin{abstract}
Kigami has shown how to construct Laplacians on certain selfsimilar fractals, first for the Sierpiński gasket and then for the class of postcritically finite (pcf) fractals, subject to the solution of certain algebraic equations. It is desirable to extend this method to as large a class of fractals as possible, so in this paper we examine a specific example that exhibits features associated with finite ramification, but which does not fall into the class of pcf fractals. We show by a method of deconstruction that this fractal is a member of a family of three fractals for which the pcf condition holds in a generalized sense. We then study the algebraic equations whose solution is required for the actual construction of the Laplacian. We obtain experimental evidence for the existence and uniqueness of solutions. This experimental work uncovers two symmetries that were not initially apparent, only one of which has a natural explanation. By exploiting the symmetries, we give a nonconstructive proof of existence.
\end{abstract}

\section{INTRODUCTION}

Analysis on fractals has been extensively developed on a restricted class of fractals. This theory, which centers around the Laplacian and its associated Dirichlet form, now includes such topics as linear and nonlinear differential equations, Brownian motion type processes, gradients and Taylor approximation, Weyl-type spectral asymptotics, and splines. See [Barlow 1998; Kigami 2001] for references. A major priority for this field is to enlarge the class of fractals that can be included. We are especially interested in methods of defining the Dirichlet form and Laplacian that are direct and constructive, in the spirit of the work of Kigami [1989; 1993] on the Sierpiński gasket and the class of postcritically finite (pcf) self-similar fractals, which includes the nested fractals of [Lindstrøm 1990]. Beyond the pcf fractals, the only other ways of obtaining Laplacians are indirect, as a biproduct of a stochastic process, and nonconstructive in that they rely on passing to a convergent subsequence. 
On the unit interval, the Laplacian is just the second derivative, and the Dirichlet form, which acts on a suitable space of functions, is given by $\mathcal{E}(u, v)=$ $\int_{0}^{1} u^{\prime}(x) v^{\prime}(x) d x$. The usual approach is to define both in terms of the first derivative, although to be precise the derivatives that enter into the Dirichlet form must be allowed to be weak derivatives, and the domain of $\mathcal{E}$ is the Sobolev space $H^{1}$. On the unit interval, functions in the domain of $\mathcal{E}$ must be continuous (but this is no longer true in higher dimensions or on manifolds, where the Dirichlet form is the integral of the length of the gradient squared). It turns out to be possible to define $\mathcal{E}$ directly by setting $\mathcal{E}(u, v)$ equal to

$\lim _{n \rightarrow \infty} n \sum_{k=1}^{n}\left(u\left(\frac{k}{n}\right)-u\left(\frac{k-1}{n}\right)\right)\left(v\left(\frac{k}{n}\right)-v\left(\frac{k-1}{n}\right)\right)$

and then to define the Laplacian via the weak formulation

$$
\int_{0}^{1}(\Delta u) v d x=-\mathcal{E}(u, v) \quad \text { if } v(0)=v(1)=0,
$$

thus bypassing the first derivative. This may seem excessive for the interval (though it does avoid a bit of functional analysis that usually is involved in the definition of Sobolev spaces), but it is absolutely essential on fractals where there is no reasonable analog of the first derivative.

In this paper we study an example that does not immediately fit into the framework of pcf fractals. We show that by performing a "deconstruction" we can bring it into a slight generalization of this framework. The real work is the construction of the Laplacian, and its associated Dirichlet form. We are able to obtain explicit formulas that involve constants solving a system of algebraic equations. The constants are determined experimentally, and may be easily computed to any desired accuracy. We also give a proof that the system of equations has a solution, but the proof is nonconstructive, relying on Brouwer's fixed point theorem. In particular, the proof does not show that the numerical values we have found are close to the values of an actual solution. The proof does not give uniqueness, although the experimental evidence suggests that the solution is unique. Moreover, the proof would not have been discovered without the experimental work, since it relies on adjoining two equations to the system expressing hidden symmetries of the problem that only became apparent upon examining the experimentally obtained numerical values.

In order to explain our results, we begin with a brief summary of the definition of pcf fractals and the results of [Kigami 1993]. We will make a few simplifying assumptions to aid the exposition. The pcf fractals $K$ we deal with will be compact subsets of some Euclidean space, and will be the attractor of an iterated function system (ifs) of contractive similarities $F_{1}, \ldots, F_{N}$, meaning

$$
K=\bigcup_{i=1}^{N} F_{i} K
$$

The main assumption is that $K$ is connected, but just barely; in particular, the image sets $F_{i} K$ may intersect only at a finite number of points. More precisely, we assume that there are a finite number of points $\left(q_{1}, \ldots, q_{m}\right)=V_{0}$, forming the boundary of $K$, such that $F_{j} q_{j}=q_{j}$ for $j \leq m$, and $F_{j} K \cap F_{k} K \subseteq$ $F_{j} V_{0} \cap F_{k} V_{0}$ for any distinct $j, k$.

A pcf fractal is in a natural way the limit of an increasing sequence of finite graphs $\Gamma_{0}, \Gamma_{1}, \ldots$ The vertices of $\Gamma_{0}$ are $V_{0}$, and in general the vertices of $\Gamma_{m}$, denoted $V_{m}$, are defined recursively by

$$
V_{m}=\bigcup_{i=1}^{N} F_{j} V_{m-1}
$$

The edge relation $x \sim_{m} y$ for $x, y \in V_{m}$ is defined by the existence of a word $w=\left(w_{1}, \ldots, w_{m}\right)$ of length $|w|=m$ on the letters $\{1, \ldots, N\}$ such that $x$ and $y$ are both in $F_{w} V_{0}$, where $F_{w}$ denotes the iterated mapping $F_{w_{1}} \circ \cdots \circ F_{w_{m}}$. Note that $\Gamma_{0}$ is just the complete graph on $V_{0}$, and $\Gamma_{m}$ reflects the connectivity structure of $K$ at resolution $m$, treating each cell $F_{w} K$ for $|w|=m$ as an undifferentiated smudge. Dirichlet forms and Laplacians on $K$ are defined as limits of Dirichlet forms and Laplacians on $\Gamma_{m}$. Functions on $K$ give rise to functions on each graph by restriction, and since $\bigcup_{m} V_{m}$ is dense in $K$, continuous functions on $K$ are uniquely determined by their restriction. The Dirichlet forms considered usually give positive capacity to points, so the domain is naturally contained within the space of continuous functions. 
We are interested in Dirichlet forms $\mathcal{E}(u, v)$, where $u$ and $v$ are real valued functions on $K$ belonging to a subspace $\operatorname{dom} \mathcal{E}$ of the continuous functions, that are self-similar in that

$$
\mathcal{E}(u, v)=\sum_{i=1}^{N} r_{i}^{-1} \mathcal{E}\left(u \circ F_{i}, v \circ F_{i}\right)
$$

for certain constants $r_{i}$ (implicit in the definition is that $\operatorname{dom} \varepsilon$ is preserved by the mappings $F_{i}$ ). We will make the further regularity assumption

$$
0<r_{i}<1 \text { for all } i \text {. }
$$

To obtain (1.2) we are more or less forced into considering a sequence of Dirichlet forms on $\Gamma_{m}$ defined inductively by

$$
\mathcal{E}_{m}(u, v)=\sum_{i=1}^{N} r_{i}^{-1} \mathcal{E}_{m-1}\left(u \circ F_{i}, v \circ F_{i}\right) .
$$

This leaves only the choice of Dirichlet form $\mathcal{E}_{0}$ on $V_{0}$,

$$
\mathcal{E}_{0}(u, v)=\sum c_{j k}\left(u\left(q_{j}\right)-u\left(q_{k}\right)\right)\left(v\left(q_{j}\right)-v\left(q_{k}\right)\right),
$$

for nonnegative conductance constants $c_{j k}$ (the reciprocals $c_{j k}^{-1}$ have the interpretation of resistance for an electrical network). We call $\mathcal{E}_{0}$ nondegenerate if enough conductances are nonzero that $\mathcal{E}_{0}(u, u)=0$ only for $u$ constant on $V_{0}$.

Not every choice of $\mathcal{E}_{0}$ will lead to a meaningful limit of $\mathcal{E}_{m}$ as $m \rightarrow \infty$. On the contrary, only a very careful balancing of the choice of $\varepsilon_{0}$ and the constants $\left\{r_{i}\right\}$ will do the trick. We require a consistency condition. If $u$ is defined on $V_{m-1}$, let $\tilde{u}$ denote the extension of $u$ to $V_{m}$ that minimizes the energy $\mathcal{E}_{m}(\tilde{u}, \tilde{u})$, called the harmonic extension (unique under the nondegeneracy condition). We require

$$
\mathcal{E}_{m}(\tilde{u}, \tilde{u})=\mathcal{E}_{m-1}(u, u) \text { for all } u .
$$

It suffices to have this condition hold for $m=1$; it follows that it holds for all $m \geq 1$. A harmonic structure is a choice of nondegenerate $\mathcal{E}_{0}$ and $\left\{r_{i}\right\}$ such that the condition holds. It is a nontrivial problem to find harmonic structures (see [Sabot 1997] for an extensive discussion of this problem). In particular, it is not kown whether every pcf fractal has a harmonic structure, and it is known that harmonic structures do not exist for every choice of $\left\{r_{i}\right\}$. Once a harmonic structure is found, it is easy to see that $\mathcal{E}_{m}(u, u)$ is an increasing function of $m$, so

$$
\mathcal{E}(u, u)=\lim _{m \rightarrow \infty} \mathcal{E}_{m}(u, u)
$$

is well-defined in $[0, \infty]$, and we can define $\operatorname{dom} \mathcal{E}$ to be the set of $u$ for which $\varepsilon(u, u)<\infty$. It is shown in [Kigami 1993] that $\operatorname{dom} \mathcal{E}$ modulo constants is a Hilbert space under the norm $\mathcal{E}(u, u)^{1 / 2}$, and dom $\mathcal{E}$ is contained in the space of continuous functions. Furthermore,

$$
\mathcal{E}(u, v)=\lim _{m \rightarrow \infty} \mathcal{E}_{m}(u, v) \quad \text { for } u, v \in \operatorname{dom} \mathcal{E}
$$

is a regular, local Dirichlet form for any reasonable measure on $K$. We consider self-similar measures $\mu$ which satisfy the identity

$$
\mu=\sum_{i=1}^{N} \mu_{i} \mu \circ F_{i}^{-1}
$$

or

$$
\int f d \mu=\sum_{i=1}^{N} \mu_{i} \int f \circ F_{i} d \mu
$$

for some probability weights $\left\{\mu_{i}\right\}$.

Given a Dirichlet form and a self-similar measure, we can define a Laplacian $\Delta$ as follows: $u \in \operatorname{dom} \Delta$ and $\Delta u=f$ if $u \in \operatorname{dom} \mathcal{E}, f$ is continuous, and

$$
\mathcal{E}(u, v)=-\int f v d \mu
$$

for every $v \in \operatorname{dom} \mathcal{E}$ vanishing on the boundary $V_{0}$. There is also a pointwise limit formula for $\Delta u$, but we will not give the general formula.

Next we discuss the deconstruction idea we will use in the study of the diamond fractal. It is well known that different choices of ifs may lead to the same fractal, and sometimes there are consequences to which choice is made. For example, the hexagasket (or fractal Star of David) shown below can

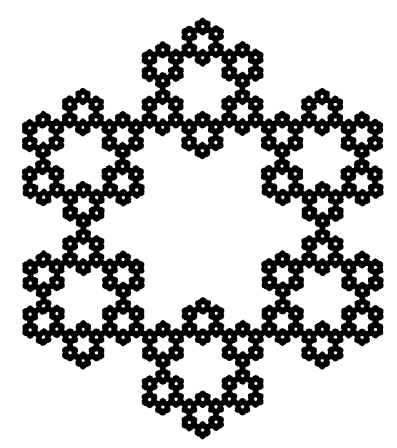


be generated by six simple homotheties with contraction ratio $\frac{1}{3}$ and fixed points the vertices of a hexagon (or star). This is a pcf fractal with boundary consisting of the six vertices. However, by including rotations in the similarities we can arrange that the boundary consist of just three of the vertices (every other one). In this case there is not a drastic difference between the two choices. In many cases, however, the new ifs must be interpreted in the more general sense of [Mauldin and Williams 1988]. Instead of a single self-similar fractal $K$ satisfying (1-1), we will have a self-similar family of fractals $K_{j}$, each of which is a union of images under contractive similarities of all the sets in the family. It is a routine matter to extend the definitions and results of [Kigami 1993] to this more general context. The point is that there are many interesting examples where the new self-similar family (containing the original fractal) with its ifs will be pcf, while the fractal with its original ifs was not.

We will refer to the process of finding a new ifs as deconstruction, in a nod to our colleagues in the humanities who have used the same term for a similar analysis of literary texts (with perhaps more questionable justifications). In fact this idea has been used many times before in the study of selfsimilar fractals, usually for the purpose of eliminating overlaps to compute dimensions (see [Edgar 1991; Strichartz et al. 1995; Strichartz and Wang 1999], for example).

Even when a deconstruction is found that restores the pcf condition, it is still necessary to find the correct conductance coefficients to construct a Dirichlet form. In this paper we carry out such a construction in one particular example, the diamond fractal. In this example the deconstruction leads to a family of three sets, which we call the diamond, the chevron and the crown. These sets possess obvious geometric symmetries, and we demand that the Dirichlet forms also possess the same symmetries. The procedure we followed in studying this example was to first use experimental methods to produce numerical approximations to the conductance constants. We then observed (by playing around with the numbers) that these constants satisfied two simple linear identities. (One of these identities can be explained as a further symmetry of the fractal, while the other awaits explanation.) By imposing these identities as a priori constraints, we were then able to give a nonconstructive proof of the existence of a nondegenerate Dirichlet form satisfying these constraints. The experimental evidence strongly supports the uniqueness of this Dirichlet form.

The success of the method of deconstruction for this example suggests that it will be possible to describe a class of fractals for which it will also work. The main problem is to find a criterion for the existence of a pcf deconstruction. The fact that it is necessary to actually construct a harmonic structure is not new, since one faces the same requirement for ordinary pcf fractals. Perhaps some of the ideas used in the existence proof for this example will be useful more generally. Hambly and Nyberg [1999] have studied self-similar Laplacians on a class of self-similar sets which allow pcf deconstructions. Under the assumption of the existence of a Laplacian, they obtained the asymptotic behavior of the eigenvalue counting function. They also obtained an existence theorem for a Laplacian, which cannot be applied to our example.

We now give a description of the diamond fractal. This is a self-similar fractal that is not pcf, generated by six contractive similarities in the plane with contraction ratio $\frac{1}{3}$, as indicated in Figure 1 . The convex hull of the fractal is a diamond (the union of two equilateral triangles), and two pairs of images of the diamond intersect along an edge. The images of the fractal in fact intersect in a Cantor set. However, the fractal appears to be finitely ramified, in that it becomes disconnected with arbitrarily small components upon the removal of suitable finite sets of points. This suggests the possibility that we can restore the pcf condition by performing a deconstruction. In fact this is possible only if we allow a slight generalizaiton of pcf to include self-similar families of sets (or "graph directed fractals" in the terminology of [Mauldin and Williams 1988]).

Thus we embed the diamond into a family of three sets, which we call the diamond, the chevron and the crown (Figure 2). The diamond is a union of two diamonds and two chevrons, the chevron is the union of two diamonds, three chevrons and one crown, while the crown is the union of four diamonds, two chevrons and four crowns. All these components intersect at isolated points, so we have a pcf structure. 


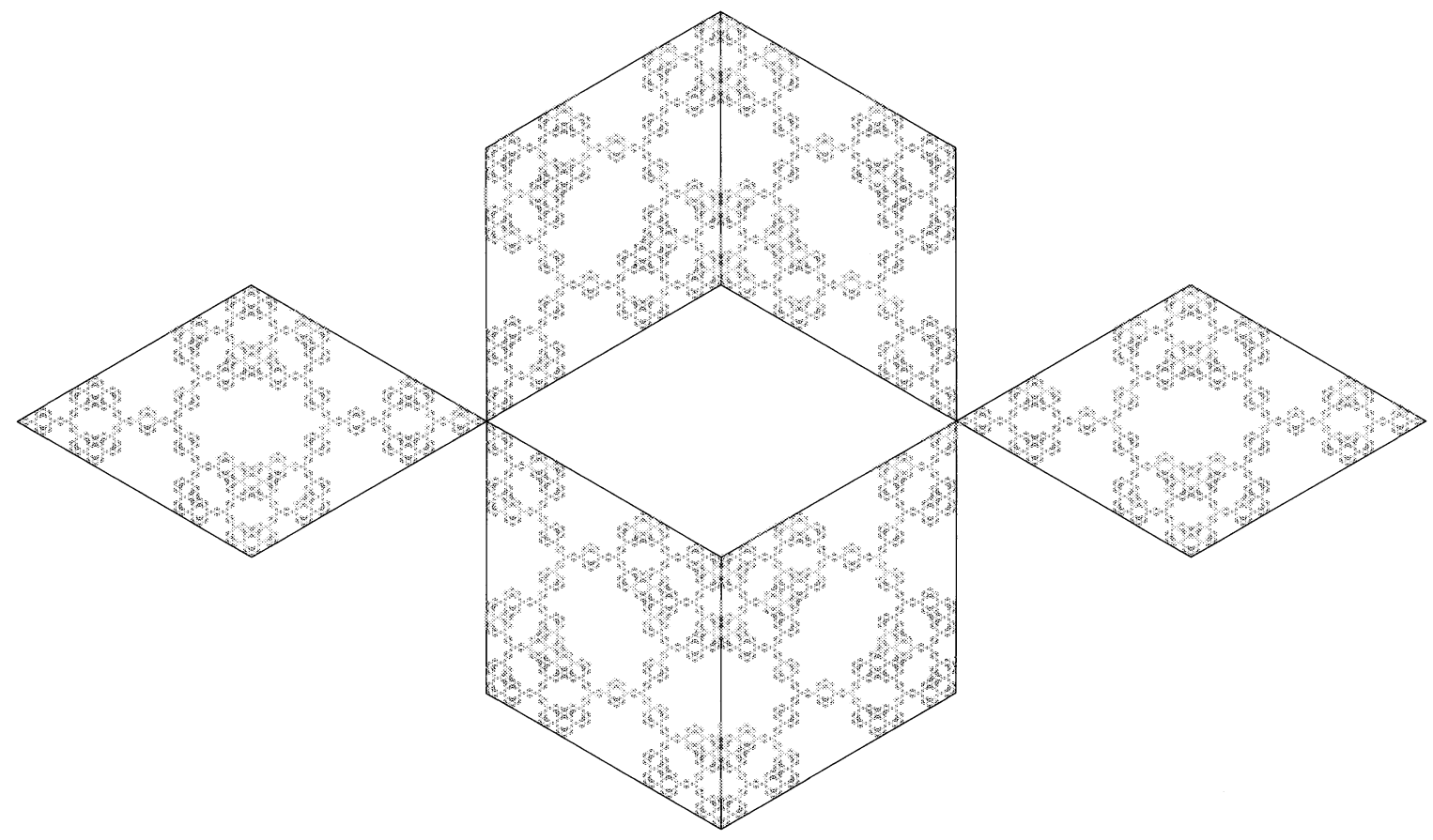

FIGURE 1. The fractal diamond and its six similarity images.

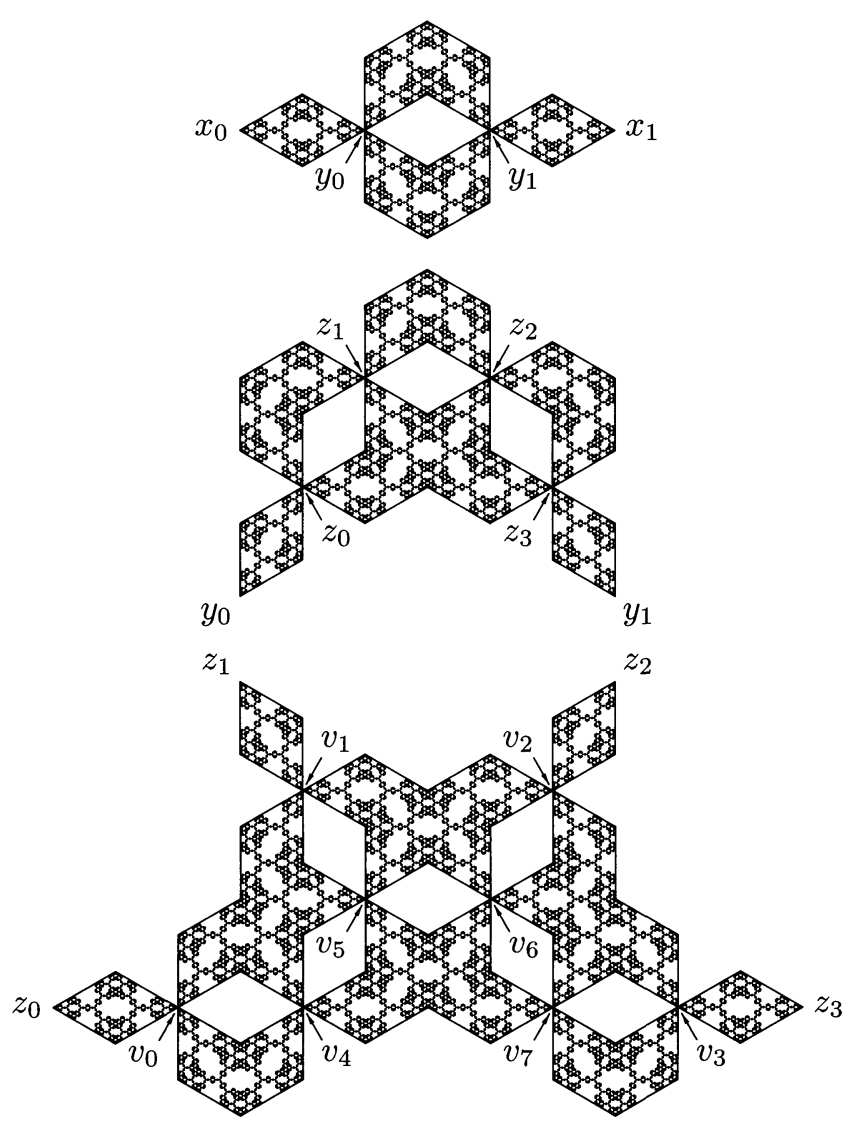

FIGURE 2. Top: the diamond. Middle: the chevron. Bottom: the crown. The decompositions are indicated, and intersection points are labeled.
The diamond and chevron have two boundary points and the crown has four (labeled $x_{0}, x_{1}$ for the diamond, $y_{0}, y_{1}$ for the chevron, and $z_{0}, z_{1}, z_{2}, z_{3}$ for the crown, in Figure 2).

\section{CONSTRUCTION OF DIRICHLET FORMS}

Our goal is to construct a self-similar family of Dirichlet forms on the diamond, chevron and crown, having all the symmetries of the fractals. We begin with Dirichlet forms on the graphs of level 0 consisting of the boundary points; abbreviating $u\left(x_{0}\right)$ as $\bar{x}_{0}$ and so on for compactness, we can write

$$
\begin{aligned}
E_{0}^{(1)}= & c_{1}\left(\bar{x}_{0}-\bar{x}_{1}\right)^{2}, \\
E_{0}^{(2)}= & c_{2}\left(\bar{y}_{0}-\bar{y}_{1}\right)^{2}, \\
E_{0}^{(3)}= & c_{3}\left(\left(\bar{z}_{0}-\bar{z}_{1}\right)^{2}+\left(\bar{z}_{2}-\bar{z}_{3}\right)^{2}\right)+c_{4}\left(\bar{z}_{1}-\bar{z}_{2}\right)^{2} \\
& +c_{5}\left(\left(\bar{z}_{0}-\bar{z}_{2}\right)^{2}+\left(\bar{z}_{1}-\bar{z}_{3}\right)^{2}\right)+c_{6}\left(\bar{z}_{0}-\bar{z}_{3}\right)^{2} .
\end{aligned}
$$

Note that we have imposed two symmetries relating the pairs $\left(z_{0}, z_{1}\right)$ and $\left(z_{2}, z_{3}\right)$, and the pairs $\left(z_{0}, z_{2}\right)$ and $\left(z_{1}, z_{3}\right)$. We may assume without loss of generality that $c_{1}=1$, since we can always achieve this by multiplying $E_{0}^{(1)}, E_{0}^{(2)}$ and $E_{0}^{(3)}$ by the same constant; this amounts to a normalization decision.

Next we assume that there is a fixed scaling factor $r$, with $0<r<1$, for comparing the Dirichlet 

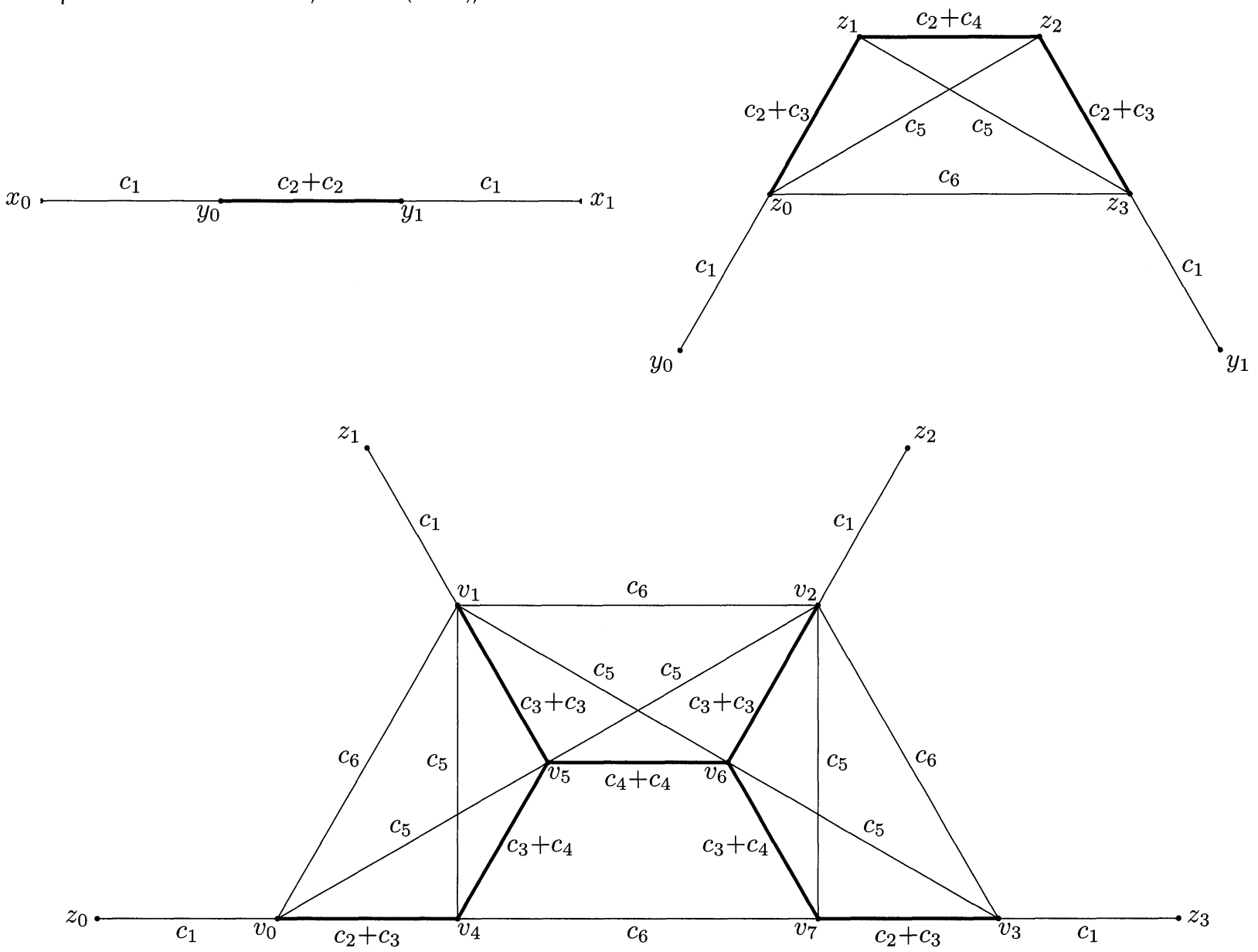

FIGURE 3. Graphs of boundary points for the first-level decomposition of the diamond, the chevron, and the crown. Thicker lines mean there are two edges between the given vertices. The labels $c_{1}, \ldots, c_{6}$ give the conductance coefficients; double edges are labeled by the sum of the conductances of the individual edges.

forms on the images of the fractals with the original. This is again a symmetry assumption, that all the sets in the decompositions have the same relative "size". We then obtain Dirichlet forms $E_{1}^{(1)}$, $E_{1}^{(2)}, E_{1}^{(3)}$ on the graphs consisting of the boundary points of the components of the first level decomposition of the diamond, chevron and crown (Figure 3 ) by summing original level 0 Dirichlet forms on the components, multiplied by $r^{-1}$. Explicitly, in the notation of Figure 3, we have

$E_{1}^{(1)}=r^{-1}\left(\left(\bar{x}_{0}-\bar{y}_{0}\right)^{2}+\left(\bar{x}_{1}-\bar{y}_{1}\right)^{2}+2 c_{2}\left(\bar{y}_{0}-\bar{y}_{1}\right)^{2}\right)$

$$
\begin{aligned}
& E_{1}^{(2)}=r^{-1}\left(\left(\bar{y}_{0}-\bar{z}_{0}\right)^{2}+\left(\bar{y}_{1}-\bar{z}_{3}\right)^{2}\right. \\
& \quad+c_{2}\left(\left(\bar{z}_{0}-\bar{z}_{1}\right)^{2}+\left(\bar{z}_{1}-\bar{z}_{2}\right)^{2}+\left(\bar{z}_{2}-\bar{z}_{3}\right)^{2}\right) \\
& \quad+c_{3}\left(\left(\bar{z}_{0}-\bar{z}_{1}\right)^{2}+\left(\bar{z}_{2}-\bar{z}_{3}\right)^{2}\right)+c_{4}\left(\bar{z}_{1}-\bar{z}_{2}\right)^{2} \\
& \left.\quad+c_{5}\left(\left(\bar{z}_{0}-\bar{z}_{2}\right)^{2}+\left(\bar{z}_{1}-\bar{z}_{3}\right)^{2}\right)+c_{6}\left(\bar{z}_{0}-\bar{z}_{3}\right)^{2}\right)
\end{aligned}
$$

and

$$
\begin{aligned}
& E_{1}^{(3)}=r^{-1}\left(\left(\bar{v}_{0}-\bar{z}_{0}\right)^{2}+\left(\bar{v}_{1}-\bar{z}_{1}\right)^{2}+\left(\bar{v}_{2}-\bar{z}_{2}\right)^{2}+\left(\bar{v}_{3}-\bar{z}_{3}\right)^{2}\right. \\
& +c_{2}\left(\left(\bar{v}_{0}-\bar{v}_{4}\right)^{2}+\left(\bar{v}_{3}-\bar{v}_{2}\right)^{2}\right) \\
& +c_{3}\left(\left(\bar{v}_{0}-\bar{v}_{4}\right)^{2}+2\left(\bar{v}_{1}-\bar{v}_{5}\right)^{2}+2\left(\bar{v}_{2}-\bar{v}_{6}\right)^{2}\right. \\
& \left.\quad+\left(\bar{v}_{3}-\bar{v}_{7}\right)^{2}+\left(\bar{v}_{4}-\bar{v}_{5}\right)^{2}+\left(\bar{v}_{6}-\bar{v}_{7}\right)^{2}\right) \\
& +c_{4}\left(\left(\bar{v}_{4}-\bar{v}_{5}\right)^{2}+2\left(\bar{v}_{5}-\bar{v}_{6}\right)^{2}+\left(\bar{v}_{6}-\bar{v}_{7}\right)^{2}\right) \\
& +c_{5}\left(\left(\bar{v}_{0}-\bar{v}_{5}\right)^{2}+\left(\bar{v}_{1}-\bar{v}_{4}\right)^{2}+\left(\bar{v}_{1}-\bar{v}_{6}\right)^{2}+\left(\bar{v}_{2}-\bar{v}_{5}\right)^{2}\right. \\
& \left.\quad+\left(\bar{v}_{2}-\bar{v}_{7}\right)^{2}+\left(\bar{v}_{3}-\bar{v}_{6}\right)^{2}+\left(\bar{v}_{4}-\bar{v}_{6}\right)^{2}+\left(\bar{v}_{5}-\bar{v}_{7}\right)^{2}\right) \\
& \left.+c_{6}\left(\left(\bar{v}_{0}-\bar{v}_{1}\right)^{2}+\left(\bar{v}_{1}-\bar{v}_{2}\right)^{2}+\left(\bar{v}_{2}-\bar{v}_{3}\right)^{2}+\left(\bar{v}_{4}-\bar{v}_{7}\right)^{2}\right)\right) .
\end{aligned}
$$

Next we define the harmonic extension to be the energy minimizer subject to the boundary values held constant. So for the diamond, we fix $\bar{x}_{0}$ and $\bar{x}_{1}$ and choose $\bar{y}_{0}$ and $\bar{y}_{1}$ to minimize $E_{1}^{(1)}$. For the chevron it is $\bar{y}_{0}$ and $\bar{y}_{1}$ that are fixed, and $\bar{z}_{j}$, $j=0,1,2,3$, that are chosen to minimize $E_{1}^{(2)}$. For 
the crown we simplified the problem by separating the even and odd parts of $u$ with respect to the reflectional symmetry. It is easy to see that the even and odd parts are orthogonal with respect to energy, so the harmonic extension may be computed by minimizing the energy of the even and odd parts separately. For an even function $\bar{z}_{3}=\bar{z}_{0}$ and $\bar{z}_{2}=\bar{z}_{1}$, so that

$$
E_{0}^{+}=\left(2 c_{3}+2 c_{5}\right)\left(\bar{z}_{0}-\bar{z}_{1}\right)^{2} .
$$

Also $\bar{v}_{2}=\bar{v}_{1}, \bar{v}_{3}=\bar{v}_{0}, \bar{v}_{6}=\bar{v}_{5}$ and $\bar{v}_{7}=\bar{v}_{4}$, so

$$
\begin{aligned}
& E_{1}^{+}=2 r^{-1}\left(\left(\bar{v}_{0}-\bar{z}_{0}\right)^{2}+\left(\bar{v}_{1}-\bar{z}_{1}\right)^{2}+c_{2}\left(\bar{v}_{0}-\bar{v}_{4}\right)^{2}\right. \\
& +c_{3}\left(\left(\bar{v}_{0}-\bar{v}_{4}\right)^{2}+2\left(\bar{v}_{1}-\bar{v}_{5}\right)^{2}+\left(\bar{v}_{4}-\bar{v}_{5}\right)^{2}\right) \\
& +c_{4}\left(\bar{v}_{4}-\bar{v}_{5}\right)^{2} \\
& +c_{5}\left(\left(\bar{v}_{0}-\bar{v}_{5}\right)^{2}+\left(\left(\bar{v}_{1}-\bar{v}_{4}\right)^{2}+\left(\bar{v}_{1}-\bar{v}_{5}\right)^{2}+\left(\bar{v}_{4}-\bar{v}_{5}\right)^{2}\right)\right) \\
& \left.+c_{6}\left(\bar{v}_{0}-\bar{v}_{1}\right)^{2}\right) .
\end{aligned}
$$

Similarly, for odd functions,

$$
E_{0}^{-}=2 c_{3}\left(\bar{z}_{0}-\bar{z}_{1}\right)^{2}+2 c_{5}\left(\bar{z}_{0}+\bar{z}_{1}\right)^{2}+4 c_{4} \bar{z}_{1}^{2}+4 c_{6} \bar{z}_{0}^{2}
$$

and

$$
\begin{aligned}
& E_{1}^{-}=2 r^{-1}\left(\left(\bar{v}_{0}-\bar{z}_{0}\right)^{2}+\left(\bar{v}_{1}-\bar{z}_{1}\right)^{2}+c_{2}\left(\bar{v}_{0}-\bar{v}_{4}\right)^{2}\right. \\
& \quad+c_{3}\left(\left(\bar{v}_{0}-\bar{v}_{4}\right)^{2}+2\left(\bar{v}_{1}-\bar{v}_{5}\right)^{2}+\left(\bar{v}_{4}-\bar{v}_{5}\right)^{2}\right) \\
& \quad+c_{4}\left(\left(\bar{v}_{4}-\bar{v}_{5}\right)^{2}+4 \bar{v}_{5}^{2}\right) \\
& \quad+c_{5}\left(\left(\bar{v}_{0}-\bar{v}_{5}\right)^{2}+\left(\bar{v}_{1}-\bar{v}_{4}\right)^{2}+\left(\bar{v}_{1}+\bar{v}_{5}\right)^{2}+\left(\bar{v}_{4}+\bar{v}_{5}\right)^{2}\right) \\
& \left.\quad+c_{6}\left(\left(\bar{v}_{0}-\bar{v}_{1}\right)^{2}+2 \bar{v}_{4}^{2}+2 \bar{v}_{1}^{2}\right)\right) .
\end{aligned}
$$

In both cases we fix $\bar{z}_{0}$ and $\bar{z}_{1}$ and choose $\bar{v}_{j}$ for $j=0,1,4,5$ to minimize $E_{1}^{+}$and $E_{1}^{-}$.

For the diamond, the minimization problem is easily solved:

$$
\bar{y}_{0}=\frac{\bar{x}_{0}+2 c_{2}\left(\bar{x}_{0}+\bar{x}_{1}\right)}{1+4 c_{2}}, \quad \bar{y}_{1}=\frac{\bar{x}_{1}+2 c_{2}\left(\bar{x}_{0}+\bar{x}_{1}\right)}{1+4 c_{2}} .
$$

The other minimizations lead to more complicated solutions involving polynomials in the $c_{j}$ coefficients. We then substitute the solutions of the harmonic extension problem into the expressions $(2-2),(2-3)$, (2-6), (2-7) for $E_{1}^{(1)}, E_{1}^{(2)}, E_{1}^{+}, E_{1}^{-}$. The resulting quadratic forms, denoted $\tilde{E}_{1}^{(1)}, \tilde{E}_{1}^{(2)}, \tilde{E}_{1}^{+}, \tilde{E}_{1}^{-}$are expressed in terms of the values of $u$ on the level 0 graph. The compatibility conditions that we need in order to have a harmonic structure are that these are equal to the original energy:

$$
\tilde{E}_{1}^{(1)}=E_{0}^{(1)}, \quad \tilde{E}_{1}^{(2)}=E_{0}^{(2)}, \quad \tilde{E}_{1}^{+}=E_{0}^{+}, \quad \tilde{E}_{1}^{-}=E_{0}^{-} .
$$

The equation $\tilde{E}_{1}^{(1)}=E_{0}^{(1)}$ is just

$$
\left(\bar{x}_{0}-\bar{x}_{1}\right)^{2}=\frac{2 c_{2}}{r\left(1+4 c_{2}\right)}\left(\bar{x}_{0}-\bar{x}_{1}\right)^{2},
$$

and this leads to the requirement

$$
r=\frac{2 c_{2}}{1+4 c_{2}} .
$$

We substitute this into the remaining three equations of $(2-9)$. The result is a system of five polynomial equations in the five coefficients $c_{2}, c_{3}, c_{4}, c_{5}$, $c_{6}$. From the numerical solutions we observed two identities relating the coefficients:

$$
\begin{aligned}
& c_{2}=c_{3}+c_{5}, \\
& c_{3}=c_{4}+c_{5}-c_{6} .
\end{aligned}
$$

In fact, we can prove that those relations are consistent with the original five polynomial equations. (There are also degenerate solutions, with some coefficients equal to zero, for which $(2-11)$ does not hold.) Now using (2-11) and (2-12), we introduce new variables $a_{1}=c_{2}, a_{2}=4 c_{4}+2 c_{2}$, and $a_{3}=$ $4 c_{5}-4 c_{3}$. Note that

$$
E_{0}^{-}=a_{2} \bar{z}_{1}^{2}+a_{3} \bar{z}_{1} \bar{z}_{0}+\left(4 c_{6}+2 c_{3}\right) \bar{z}_{0}^{2} .
$$

To obtain a set of equations for $\left(a_{1}, a_{2}, a_{3}\right)$ equivalent to the equations for the $c_{j}$ coefficients, first let $\bar{y}_{0}=\frac{1}{2}, \bar{y}_{1}=-\frac{1}{2}, \bar{z}_{0}=\frac{1}{2} p, \bar{z}_{1}=\frac{1}{2} q, \bar{z}_{2}=-\frac{1}{2} q$ and $\bar{z}_{3}=-\frac{1}{2} p$ in $(2-3)$. (This is the odd part of $E_{1}^{(2)}$.) This polynomial of $p$ and $q$ is denoted by $Q_{1}(p, q)$. Next, let $\bar{z}_{0}=b, \bar{z}_{1}=a, \bar{v}_{0}=z, \bar{v}_{1}=x, \bar{v}_{4}=w$, and $\bar{v}_{5}=y$ in $E_{1}^{-}$. This polynomial of $a, b, x, y, z, w$ is denoted by $Q_{2}(a, b, x, y, z, w)$. Set

$$
Q_{3}(a, b)=\min _{x, y, z, w} Q_{2}(a, b, x, y, z, w) .
$$

Define a map $R: \mathbb{R}^{3} \rightarrow \mathbb{R}^{3}$ with coordinate functions

$$
\begin{aligned}
& R_{1}\left(a_{1}, a_{2}, a_{3}\right)=\min _{p, q} Q_{1}(p, q), \\
& R_{2}\left(a_{1}, a_{2}, a_{3}\right)=\frac{1}{2} \frac{\partial^{2} Q_{3}}{\partial a^{2}} \\
& R_{3}\left(a_{1}, a_{2}, a_{3}\right)=\frac{\partial^{2} Q_{3}}{\partial a \partial b} .
\end{aligned}
$$

Then the equations are simply that $\left(a_{1}, a_{2}, a_{3}\right)$ is a fixed point of $R$. They are too complicated to solve algebraically, but can be solved numerically by using the iteration of the map $R$. We used Maple $\mathrm{V}$ to 
compute $R$ symbolically and iterate $R$ numerically, and found

$$
\begin{aligned}
c_{2} & =0.87433356450232153555 \\
c_{3} & =0.51869210797723658737 \\
c_{4} & =0.47199053889709250095 \\
c_{5} & =0.35564145652508494819 \\
c_{6} & =0.30893988744494086177 \\
r & =0.38882302908449559258
\end{aligned}
$$

Since these equations are of fixed point form, we tried iterating from many different starting values, and the iterations converged rapidly to this solution in all cases. Thus we have some experimental evidence that the system has a unique positive solution. Later we will give a proof of existence.

\section{HARMONIC FUNCTIONS}

With the determination of the constants given by (2-13) we can give explicit solutions to the harmonic extension algorithm. For the diamond we have

$$
\begin{aligned}
& \bar{y}_{0}=(1-r) \bar{x}_{0}+r \bar{x}_{1}, \\
& \bar{y}_{1}=r \bar{x}_{0}+(1-r) \bar{x}_{1},
\end{aligned}
$$

as can be seen from $(2-8)$ and $(2-10)$. For the chevron the answer turns out to be

$$
\begin{aligned}
& \bar{z}_{0}=d_{1} \bar{y}_{0}+\left(1-d_{1}\right) \bar{y}_{1}, \\
& \bar{z}_{1}=d_{2} \bar{y}_{0}+\left(1-d_{2}\right) \bar{y}_{1}, \\
& \bar{z}_{2}=\left(1-d_{2}\right) \bar{y}_{0}+d_{2} \bar{y}_{1}, \\
& \bar{z}_{3}=\left(1-d_{1}\right) \bar{y}_{0}+d_{1} \bar{y}_{1},
\end{aligned}
$$

where $d_{1}=0.6600389747$ and $d_{2}=0.5373812469$. The form of the solution is clear by symmetry and the fact that constant functions are harmonic, so it is the computation of the coefficients $d_{1}$ and $d_{2}$ that requires the specific values in (2-13). For the crown the solution is given by

$$
\begin{aligned}
& \bar{v}_{0}=b_{0} \bar{z}_{0}+b_{1} \bar{z}_{1}+b_{2} \bar{z}_{2}+b_{3} \bar{z}_{3}, \\
& \bar{v}_{1}=b_{1} \bar{z}_{0}+b_{4} \bar{z}_{1}+b_{5} \bar{z}_{2}+b_{2} \bar{z}_{3}, \\
& \bar{v}_{2}=b_{2} \bar{z}_{0}+b_{5} \bar{z}_{1}+b_{4} \bar{z}_{2}+b_{1} \bar{z}_{3}, \\
& \bar{v}_{3}=b_{3} \bar{z}_{0}+b_{2} \bar{z}_{1}+b_{1} \bar{z}_{2}+b_{0} \bar{z}_{3}, \\
& \bar{v}_{4}=b_{6} \bar{z}_{0}+b_{7} \bar{z}_{1}+b_{8} \bar{z}_{2}+b_{9} \bar{z}_{3}, \\
& \bar{v}_{5}=b_{7} \bar{z}_{0}+b_{10} \bar{z}_{1}+b_{11} \bar{z}_{2}+b_{8} \bar{z}_{3}, \\
& \bar{v}_{6}=b_{8} \bar{z}_{0}+b_{11} \bar{z}_{1}+b_{10} \bar{z}_{2}+b_{7} \bar{z}_{3}, \\
& \bar{v}_{7}=b_{9} \bar{z}_{0}+b_{8} \bar{z}_{1}+b_{7} \bar{z}_{2}+b_{6} \bar{z}_{3},
\end{aligned}
$$

where

$$
\begin{array}{ll}
b_{0}=0.5399160321, & b_{1}=0.2016794364, \\
b_{2}=0.1382815883, & b_{3}=0.1201229427, \\
b_{4}=0.4765181840, & b_{5}=0.1835207909, \\
b_{6}=0.3556777973, & b_{7}=0.2617128040, \\
b_{8}=0.2009059485, & b_{9}=0.1817034495, \\
b_{10}=0.2948709419, & b_{11}=0.2425103052 .
\end{array}
$$

The $b_{j}$ satisfy certain identities. From the fact that constants are harmonic we obtain

$$
\begin{array}{ll}
b_{0}+b_{1}+b_{2}+b_{3}=1, & b_{1}+b_{2}+b_{4}+b_{5}=1, \\
b_{6}+b_{7}+b_{8}+b_{9}=1, & b_{7}+b_{8}+b_{10}+b_{11}=1,
\end{array}
$$

which imply $b_{0}-b_{4}=b_{5}-b_{3}$ and $b_{6}-b_{10}=b_{11}-b_{9}$. But in fact

$$
\begin{aligned}
& b_{0}-b_{4}=b_{5}-b_{3}=b_{1}-b_{2}, \\
& b_{6}-b_{10}=b_{11}-b_{9}=b_{7}-b_{8} .
\end{aligned}
$$

Also there are identities

$$
d_{1}=b_{0}+b_{1}, \quad d_{2}=b_{6}+b_{9}
$$

relating the coefficients in (3-2) and (3-3).

We can give a simple explanation for the identities $(2-11)$ and (3-4). The crown is composed of two copies of the chevron joined along the line of symmetry. If $u$ is an even function on the crown, we can regard the restriction $\tilde{u}$ to either half as a function on the chevron, and so obtain a one-toone correspondence between even continuous functions on the crown and continuous functions on the chevron. The symmetry condition we want is that the energy of $u$ should be twice the energy of $\tilde{u}$. At level 0 we have $E_{0}^{(2)}(\tilde{u})=c_{2}\left(\tilde{u}\left(y_{0}\right)-\tilde{u}\left(y_{1}\right)\right)^{2}$ and $E_{0}^{+}(u)=\left(2 c_{3}+2 c_{5}\right)\left(u\left(z_{0}\right)-u\left(z_{1}\right)\right)^{2}$ by $(2-1)$ and $(2-5)$. But $\tilde{u}\left(y_{0}\right)=u\left(z_{0}\right)$ and $\tilde{u}\left(y_{1}\right)=u\left(z_{1}\right)$ under the correspondence, so we obtain $(2-11)$ from $E_{0}^{+}(u)=2 E_{0}^{(2)}(\tilde{u})$. It is clear from the subdivision scheme that the same identity persists at all levels. In particular, $u$ is harmonic on the crown if and only if $\tilde{u}$ is harmonic on the chevron. This means that (3-3) for even functions msut be the same as (3-2) under the correspondence $\left(\bar{z}_{0}=\bar{z}_{3}, \bar{z}_{1}=\bar{z}_{2}\right.$, $\left.\bar{v}_{0}=\bar{v}_{3}, \bar{v}_{1}=\bar{v}_{4}, \bar{v}_{2}=\bar{v}_{7}, \bar{v}_{5}=\bar{v}_{6}\right) \leftrightarrow\left(\tilde{u}\left(y_{0}\right), \tilde{u}\left(y_{1}\right)\right.$, $\left.\tilde{u}\left(z_{0}\right), \tilde{u}\left(z_{3}\right), \tilde{u}\left(z_{1}\right), \tilde{u}\left(z_{2}\right)\right)$. This yields (3-4).

Figure 4 shows the graph of a harmonic function on the diamond, obtained by recursive application of $(3-1)-(3-3)$. Modulo constants, the space of harmonic functions on the diamond is one dimensional, 


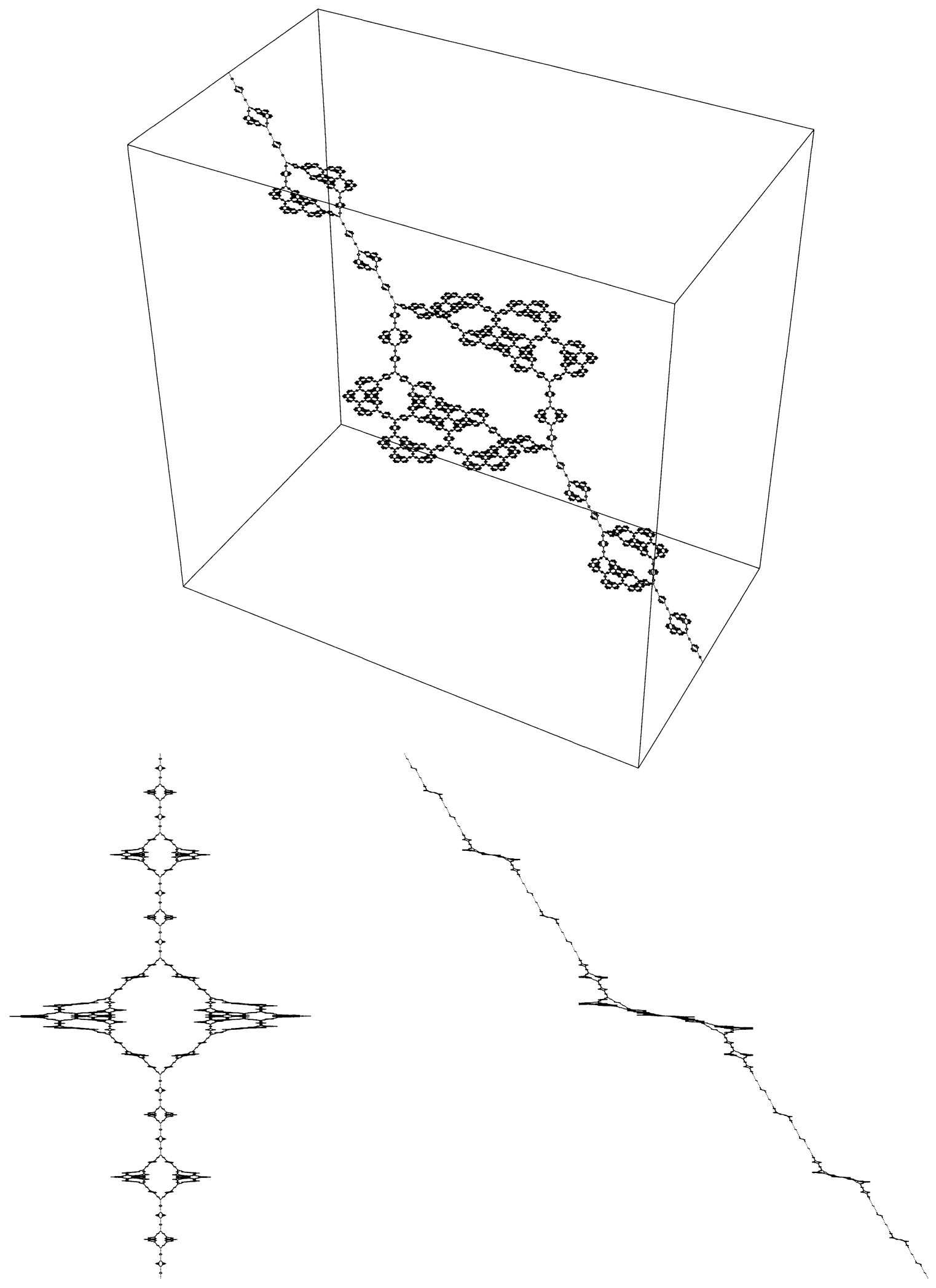

FIGURE 4. The graph of a harmonic function on the diamond. Bottom left: projection onto the left wall of the box. Bottom right: projection onto the back wall. 
so this is essentially the general harmonic function. Paradoxically, the space of harmonic functions on the crown is 4 dimensional, but on any particular crown in the diamond, there is only a 2 dimensional subspace (containing the constants) of restrictions of global harmonic functions. By looking at differently located crowns one can eventually obtain functions spanning the entire 4 dimensional space. A similar situation also arises in many pcf fractals as well, such as the hexagasket.

\section{EXISTENCE PROOF}

In this section we give an existence proof for positive solutions of the 3 equations $R\left(a_{1}, a_{2}, a_{3}\right)=$ $\left(a_{1}, a_{2}, a_{3}\right)$. Before doing this, we note that there is a degenerate solution to the original system of equations with $c_{3}=c_{4}=c_{5}=c_{6}=0$, but $(2-11)$ does not hold. In effect this makes the energy on the crown identically zero, so that all paths through crowns are blocked. However, it is clear from Figure 2 or 3 that there are still two paths joining $x_{0}$ to $x_{1}$ in the diamond, going along the upper and lower boundaries, and a single path joining $y_{0}$ to $y_{1}$ in the chevron, going along the upper boundary, that never pass through a crown. In place of $(2-3)$ we have

$$
\begin{aligned}
E_{1}^{(2)}= & r^{-1}\left(\left(\bar{y}_{0}-\bar{z}_{0}\right)^{2}+\left(\bar{y}_{1}-\bar{z}_{3}\right)^{2}\right. \\
& \left.+c_{2}\left(\left(\bar{z}_{0}-\bar{z}_{1}\right)^{2}+\left(\bar{z}_{1}-\bar{z}_{2}\right)^{2}+\left(\bar{z}_{2}-\bar{z}_{3}\right)^{2}\right)\right) .
\end{aligned}
$$

Minimizing this energy with $\bar{y}_{0}$ and $\bar{y}_{1}$ fixed yields

$$
\bar{z}_{0}-\bar{z}_{1}=\bar{z}_{1}-\bar{z}_{2}=\bar{z}_{2}-\bar{z}_{3}=\frac{1}{3}\left(\bar{z}_{0}-\bar{z}_{3}\right)
$$

and

$$
\bar{z}_{0}-\bar{z}_{3}=\left(1+\frac{2}{3} c_{2}\right)^{-1}\left(\bar{y}_{0}-\bar{y}_{1}\right),
$$

and so we find

$$
\begin{aligned}
E_{1}^{(2)} & =\frac{1}{r}\left(\frac{1}{2}\left(1-\frac{1}{1+\frac{2}{3} c_{2}}\right)^{2}+\frac{c_{2}}{3}\left(\frac{1}{1+\frac{2}{3} c_{2}}\right)^{2}\right)\left(\bar{y}_{0}-\bar{y}_{1}\right)^{2} \\
& =\frac{1+4 c_{2}}{6+4 c_{2}}\left(\bar{y}_{0}-\bar{y}_{1}\right)^{2} .
\end{aligned}
$$

where we have used (2-10) for $r$. Setting $E_{1}^{(2)}=E_{0}^{(2)}$ we obtain a quadratic equation for $c_{2}$ with $c_{2}=$ $\frac{1}{4}(\sqrt{5}-1)$ as the positive solution. (There is also another degenerate solution with $c_{2}=c_{3}$ and $c_{4}=$ $c_{5}=c_{6}=0$ but we will not describe it here.)

To prove the existence of nondegenerate solutions, we will use the method of [Lindstrøm 1990], which involves applying the Brouwer fixed point theorem.
The difficulty is to obtain bounds for all the constants, both above and below (away from zero). For example, we can easily modify the computation of the degenerate solution just given to prove the a priori lower bound

$$
c_{2} \geq \frac{1}{4}(\sqrt{5}-1)
$$

for any solution. Observe that the right side of (4-1) is always a lower bound for $E_{1}^{(2)}$. When we minimize $E_{1}^{(2)}$ given by (2-3) rather than (4-1) we will get different values for $\bar{z}_{j}$, but they will produce values for the right side of $(4-1)$ that are at least as large as the minimum, the right side of (4-2), that we just found. That means that (4-2) bcomes an inequality $(\geq)$, and since $E_{1}^{(2)}=E_{0}^{(2)}$ for the solution, we obtain (using (2-10) for $r$ ),

$$
\frac{2 c_{2}^{2}}{1+4 c_{2}} \geq \frac{1}{2}\left(1-\frac{1}{1+\frac{2}{3} c_{2}}\right)^{2}+\frac{c_{2}}{3}\left(\frac{1}{1+\frac{2}{3} c_{2}}\right)^{2}
$$

which simplifies to

$$
4 c_{2}^{2}+2 c_{2}-1 \geq 0
$$

and this requires $(4-3)$.

From (2-10) we can obtain estimates for $r$, namely

$$
\frac{1}{10}(5-\sqrt{5}) \leq r \leq \frac{1}{2}
$$

the lower bound coming from the lower bound for $c_{2}$ in (4-3), and the upper bound coming from the limit as $c_{2} \rightarrow \infty$.

To obtain an upper bound for $c_{2}$ we consider an odd function on the chevron, with $\bar{y}_{0}=1$ and $\bar{y}_{1}=$ -1 to be specific, so $E_{0}^{(2)}=4 c_{2}$. Since $E_{1}^{(2)}=4 c_{2}$ minimizes $(2-3)$, it is bounded above by the right side of $(2-3)$ with the choice $\bar{z}_{j}=0$ for $j=0,1,2,3$. This yields $4 c_{2} \leq 2 r^{-1}$, and using (2-10), $4 c_{2}^{2}-4 c_{2}-$ $1 \leq 0$, or

$$
c_{2} \leq \frac{1}{2}(1+\sqrt{2})
$$

We apply similar reasoning to an odd function on the crown with $\bar{z}_{0}=\bar{z}_{1}=1$ so $E_{0}^{-}=4\left(c_{4}+2 c_{5}+c_{6}\right)$, and we bound $E_{1}^{-}$from above by choosing $\bar{v}_{j}=0$ for all $j$ on the right side of (2.10). We obtain $4\left(c_{4}+\right.$ $\left.2 c_{5}+c_{6}\right) \leq 4 r^{-1}$ hence

$$
c_{4}+2 c_{5}+c_{6} \leq \frac{10}{5-\sqrt{5}}
$$

using the lower bound in (4-4). This suffices to bound from above all coefficients in view of $(2-11)$. 
Now we can give the Brouwer fixed point theorem argument. Consider the original system of 5 equations in $c_{2}, c_{3}, c_{4}, c_{5}, c_{6}$, assuming $r$ is given by $(2-10)$. We adjoin the identities $(2-11)$ and $(2-12)$ by considering the three-dimensional subspace $V$ of $\mathbb{R}^{5}$ defined by these identities. Within $V$ we consider the set $P$ defined by the inequalities (4-3), $(4-5),(4-6)$ and $c_{j} \geq 0$ for all $j$. It is easy to see that $P$ is topologically a closed ball. Our equations have the form

$$
c=\Phi(c)
$$

for a continuous function $\Phi$, so we need to check that $\Phi$ maps $P$ to $P$. We have already observed that $\Phi$ maps $V$ to $V$, and it is clear that the nonnegativity of coefficients is preserved. It suffices to show that the estimates (4-3), (4-5) and (4-6) are preserved under $\Phi$. Note that we proved these estimates under the assumption (4-7), whereas now we need to show that if they hold for $c$ then they hold for $c^{\prime}=\Phi(c)$. For example, the argument for (4-3) yields instead

$$
c_{2}^{\prime} \geq \frac{1+4 c_{2}}{6+4 c_{2}} .
$$

But since the right side of (4-8) is an increasing function of $c_{2}$ it assumes its minimum value at the lower bound in (4-3), which gives the same lower bound for $c_{2}^{\prime}$. Similar arguments show that the upper bounds are preserved.

The Brouwer fixed point theorem gives (nonconstructively) the existence of a solution to (4-7) in $P$. This is a nondegenerate structure in that $c_{2}$ is positive, and so are some of the $c_{j}$ 's for $j=3,4,5,6$, because of $(2-11)$ and $(2-12)$. In fact these identities require at least one of the pairs $\left(c_{3}, c_{4}\right),\left(c_{3}, c_{5}\right)$ or $\left(c_{5}, c_{6}\right)$ to be both positive. It is not difficult to see that this requires all $c_{j}$ 's to be positive, because we can join any pair of boundary points $\left(z_{j}, z_{k}\right)$ of the crown by a path that only uses edges associated with the positive pairs. For example, if the pair $\left(c_{5}, c_{6}\right)$ is positive, then $z_{0}, z_{3}, z_{1}$ is such a path joining $z_{0}$ to $z_{1}$, and $z_{1}, z_{3}, z_{0}, z_{2}$ is such a path joining $z_{1}$ to $z_{2}$.

It is possible that the method of [Sabot 1997] could be used to prove uniqueness of nondegenerate solutions.

\section{ACKNOWLEDGEMENTS}

We are grateful to Alexander Teplyaev and Silvio Levy for suggestions and technical assistance with the figures.

\section{REFERENCES}

[Barlow 1998] M. T. Barlow, "Diffusions on fractals", pp. 1-121 in Lectures on probability theory and statistics (Saint-Flour, 1995), edited by P. Bernard, Lecture Notes in Math. 1690, Springer, Berlin, 1998.

[Edgar 1991] G. A. Edgar, "A fractal puzzle", Math. Intelligencer 13:3 (1991), 44-50.

[Hambly and Nyberg 1999] B. Hambly and Nyberg, "Finitely ramified graph directed fractals, spectral asymptotics and the multidimensional renewal theorem", technical report 1999-12, BRIMS (HewlettPackard Labs), 1999. See http://www.hpl.hp.com/ techreports/1999/HPL-BRIMS-1999-12.html.

[Kigami 1989] J. Kigami, "A harmonic calculus on the Sierpiński spaces", Japan J. Appl. Math. 6:2 (1989), 259-290.

[Kigami 1993] J. Kigami, "Harmonic calculus on p.c.f. self-similar sets", Trans. Amer. Math. Soc. 335:2 (1993), 721-755.

[Kigami 2001] J. Kigami, Analysis on fractals, Cambridge tracts in math. 143, Cambridge Univ. Press, New York, 2001.

[Lindstrøm 1990] T. Lindstrøm, Brownian motion on nested fractals, Mem. Amer. Math. Soc. 420, Amer. Math. Soc., Providence, RI, 1990.

[Mauldin and Williams 1988] R. D. Mauldin and S. C. Williams, "Hausdorff dimension in graph directed constructions", Trans. Amer. Math. Soc. 309:2 (1988), 811-829.

[Sabot 1997] C. Sabot, "Existence and uniqueness of diffusions on finitely ramified self-similar fractals", Ann. Sci. École Norm. Sup. (4) 30:5 (1997), 605-673.

[Strichartz and Wang 1999] R. S. Strichartz and Y. Wang, "Geometry of self-affine tiles, I", Indiana Univ. Math. J. 48:1 (1999), 1-20.

[Strichartz et al. 1995] R. S. Strichartz, A. Taylor, and T. Zhang, "Densities of self-similar measures on the line", Experiment. Math. 4:2 (1995), 101-128. 
Jun Kigami, Department of Applied Analysis and Complex Dynamical Systems, Graduate School of Informatics, Kyoto University, Kyoto 606-8501, Japan (kigami@i.kyoto-u.ac.jp)

Robert S. Strichartz, Mathematics Department, Malott Hall, Cornell University, Ithaca, NY 14853, United States (str@math.cornell.edu)

Katharine C. Walker, Mathematics Department, Yale University, New Haven, CT 06520, United States. Current address: Mathematics Department, University of Chicago, Chicago, IL 60637, United States (kwalker@math.uchicago.edu, kacey629@yahoo.com)

Received October 27, 2000; accepted in revised from February 20, 2001 